\title{
Effect of Moderate Intensity Aerobic Exercise on Migraine Headache Indexes and Quality of Life Improvement in Women with Migraine
}

\author{
Pairo Z.. ${ }^{1}$ SSc, Parnow A.* PhD, Sari-Aslani P. ${ }^{2}$ PhD, Eslami R. ${ }^{3}$ PhD
}

\begin{abstract}
*Sport Sciences Department, Sport Sciences Faculty, Razi University, Kermanshah, Iran 1 Sport Sciences Department, Sport Sciences Faculty, Razi University, Kermanshah, Iran 2Neurology Department, Medicine Faculty, University of Medical Sciences, Kermanshah, Iran ${ }^{3}$ Exercise Physiology Department, Physical Education \& Sport Sciences Faculty, Allame Tabatabaei University, Tehran, Iran
\end{abstract}

\begin{abstract}
Aims: The beneficial effects of physical activities on migraine indices, i. e. frequency, duration, and intensity of the attacks, have been proved by different studies. Despite the fact, it is required to notice the sport characteristics, such as intensity, duration, repetition, and type of the sport, to investigate the beneficial effects of the physical activities on migraine treatment. The aim of this study was to investigate the effects of the Moderate Intensity Aerobic (MIA) exercises on the migraine indices and quality of life in women with migraine.

Materials \& Methods: In the controlled random semi-experimental study, 20 non-athlete women with migraine were studied in Kermanshah in 2015. The subjects were divided into two groups including control and MIA groups ( $n=10$ per group). 8-weak MIA exercises (13-15 Borg RPE Scale) consisted of three 40minute sessions a weak. The migraine indices of the subjects (frequency, duration, and intensity) were measured one month before and after the exercises. In addition, the quality of life and the aerobic power of the subjects were assessed 48 hours before and after the exercises. Data was analyzed by SPSS 21 software using ANCOVA, LSD post-hoc, and independent T tests.

Findings: MIA exercises resulted in significant reductions in the intensity, frequency, and duration of headache attacks, as well as an increase in the aerobic power $(\mathrm{p}<0.001)$. Passing from pretest to posttest, the variable change percentage was significant between control and exercise groups $(\mathrm{p}<0.05)$.

Conclusion: MIA exercises lead to a reduction in the migraine headache, as well as an improvement in the quality of life and aerobic readiness, in the women with migraine.
\end{abstract}

\section{Keywords}

Headache [https://www.ncbi.nlm.nih.gov/mesh/68006261];

Migraine Disorders [https://www.ncbi.nlm.nih.gov/mesh/68008881];

Women [https://www.ncbi.nlm.nih.gov/mesh/68014930];

Aerobic Exercises [https://www.ncbi.nlm.nih.gov/mesh/68015444]

*Corresponding Author

Tel: $+98(83) 34283275$

Fax: $+98(83) 34274585$

Address: Sport Sciences Faculty, Razi University, Daneshgah Street, Kermanshah, Iran. Postal Code: 6714414874

parnowabdolhossein@gmail.com

Received: November 28, $2015 \quad$ Accepted: May 10, $2016 \quad$ ePublished: October 1, 2016 


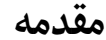

ميكَرن يك اختلال عصبى است كه در اثر مجموعهاى از وقايع در

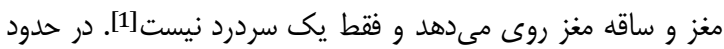
Tو\% سردردها بلهنوان سردردهاى نوع تنشى (TTH) و ميكرن

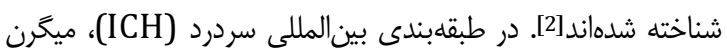

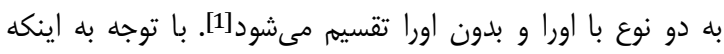

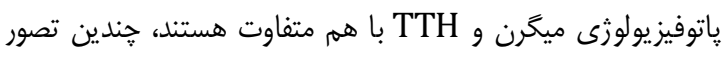

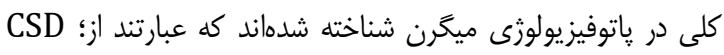

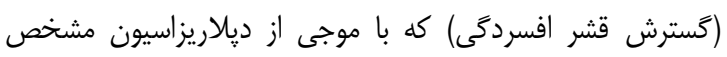

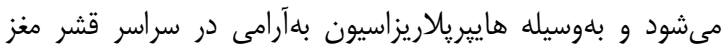

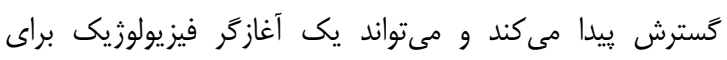

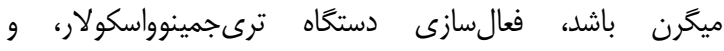
حساسسازى مركزى و محيطى درد[3].

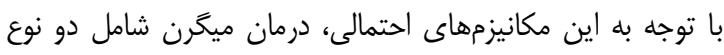

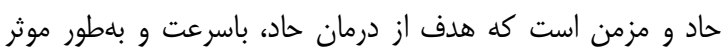

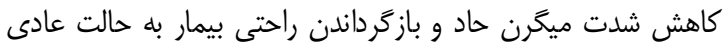

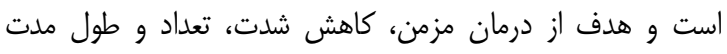

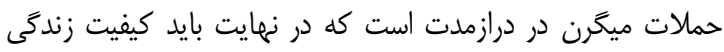

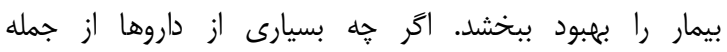
سديمناير كسن، تنگ كنندهاى عروقى مانى مانند كافئين و ترييتانها

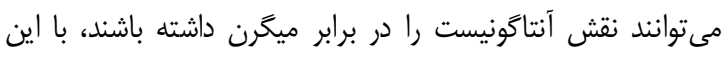

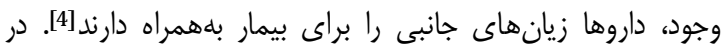

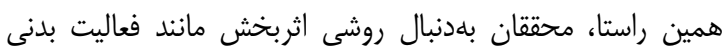

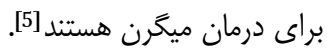
ورزش يكى فعاليت بدنى منظه، هدفمند و تكرارى است كه به با هدف

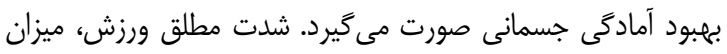

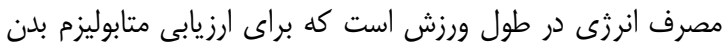

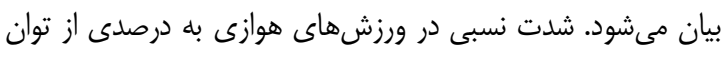

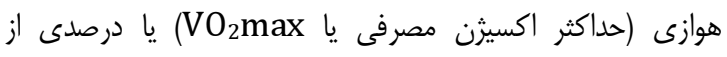

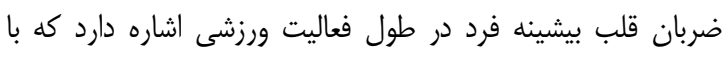
توجه به وجود ارتباط بين VO

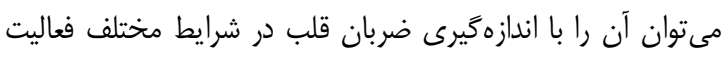

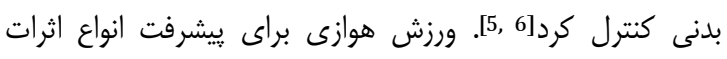

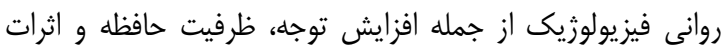

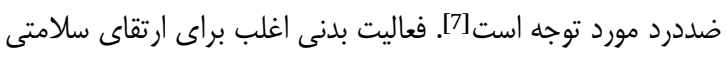

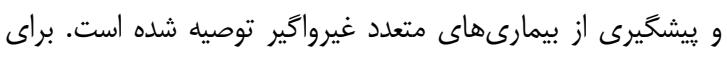

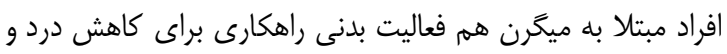

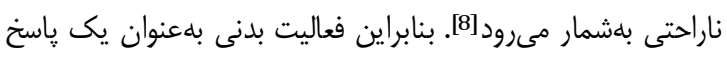

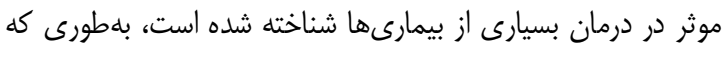

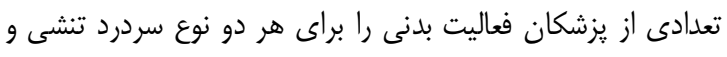

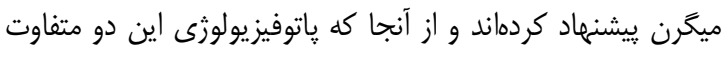

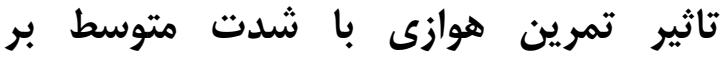 شاخصهاى سردرد ميكرنى و بهبود كيفيت زندكَى در زنان ميگَرنى}

زهرا يبيرو MSc

كروه علوم ورزشى، دانشكده علوم ورزشى، دانشخاه رازى، كرمانشاه، ايران PhD عبدالحسين يرنو كروه علوم ورزشى، دانشكده علوم ورزشى، دانشخاه رازى، كرمانشاه، ايران

ييام سارى اصلانى PhD

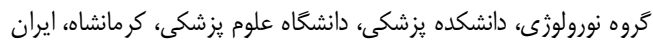

PhD رسول اسلامى

كروه فيزيولوزى ورزشى، دانشكده تربيت بدنى و علوم ورزشى، دانشعاه علامه طباطبايى، تهران، ايران

اهذاف: در مطالعات مختلف، تاثيرات سودمند فعاليت بدنى بر ماتر

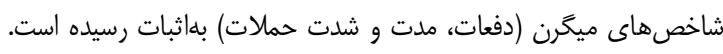

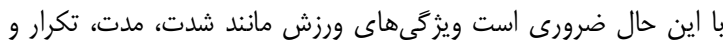

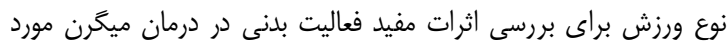

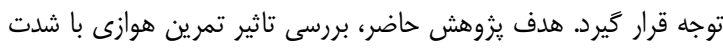

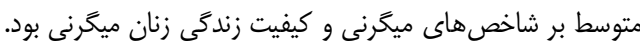

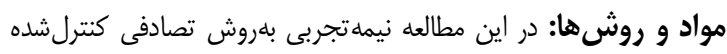

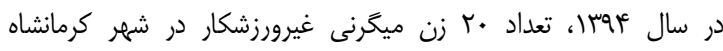

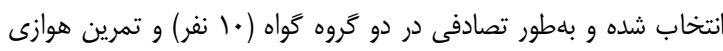

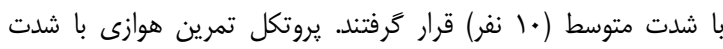

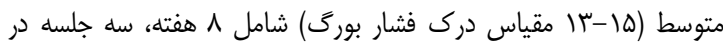

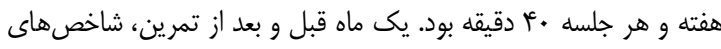

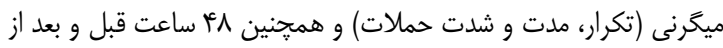

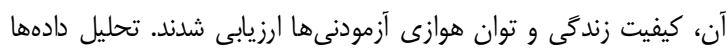

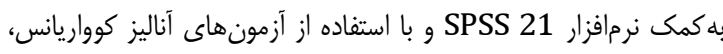
تعقيبى LSD و T Tستقل انجام شد.

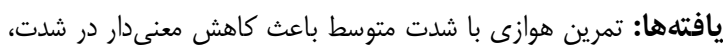

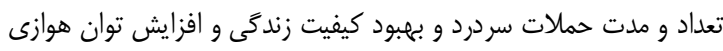

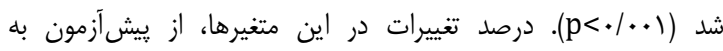

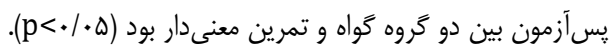

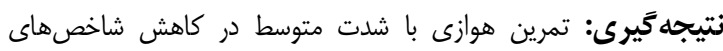

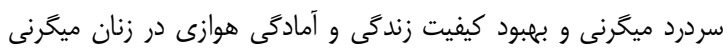
موثر است. كليدوازهها: سردرد، ميكرن، زنان، تمرين هوازى

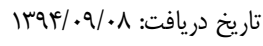

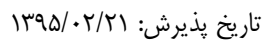
"نويسنده مسئول: parnowabdolhossein@gmail.com 


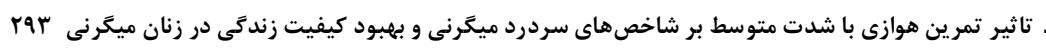

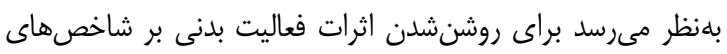

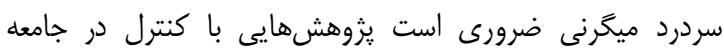

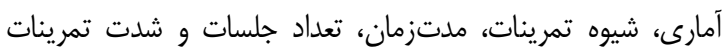

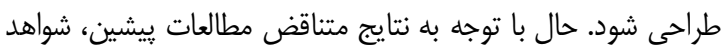

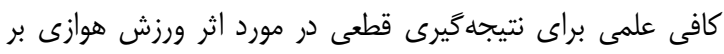

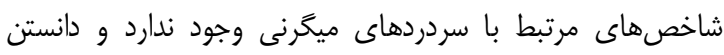

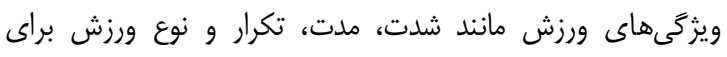

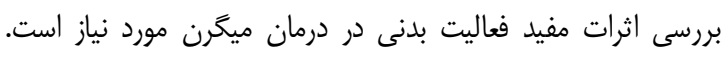

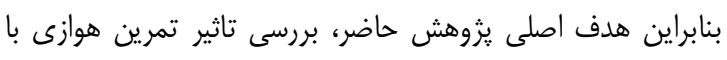

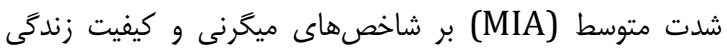

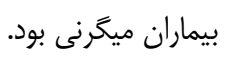

\section{مواد و روشها}

اين مطالعه نيمهتجربى و كاربردى، يك كاردار آزمايى بالينى بهروش

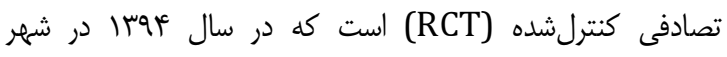

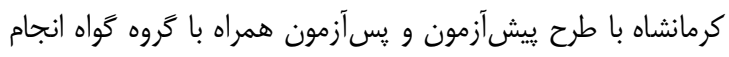

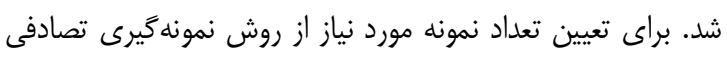

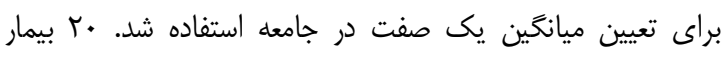

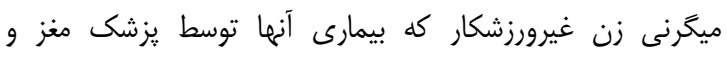

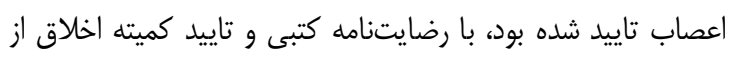

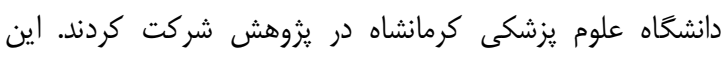

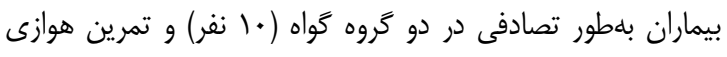

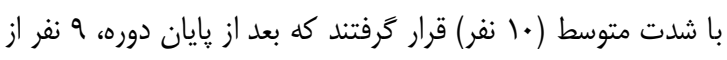

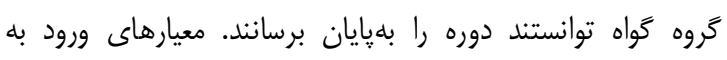

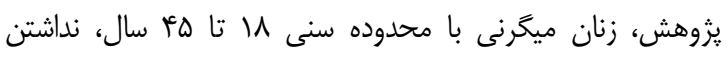

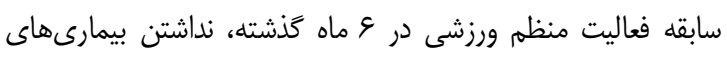

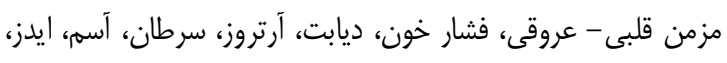

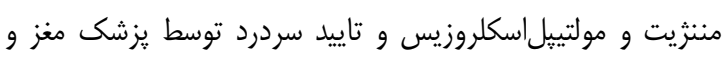

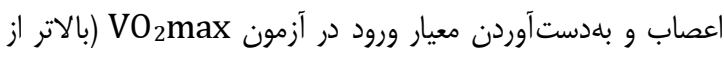

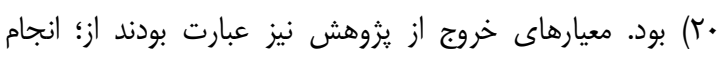

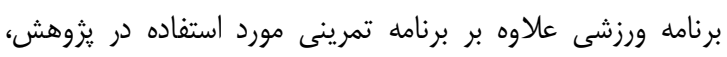

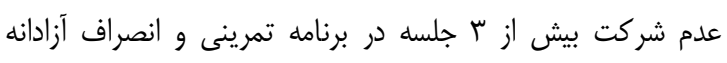

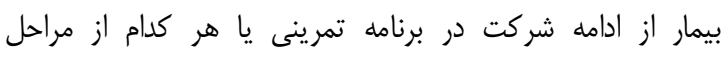

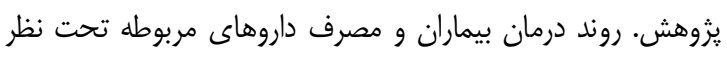

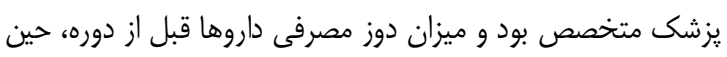
و بعد از دوره ثبت شد.

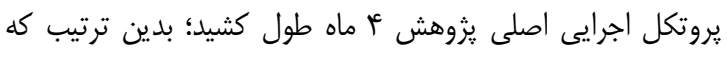

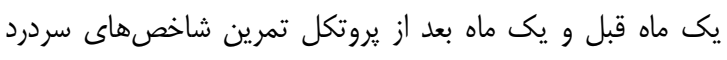

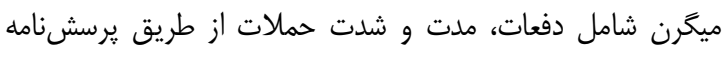

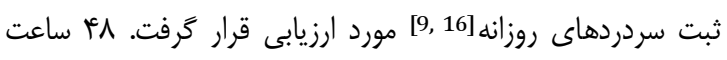

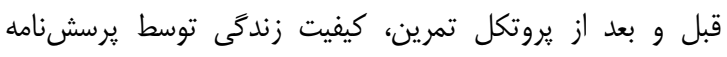

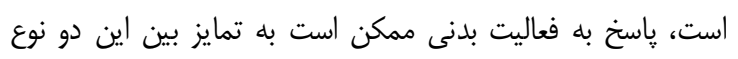
سردرد كمى كند[5].

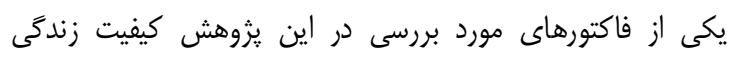

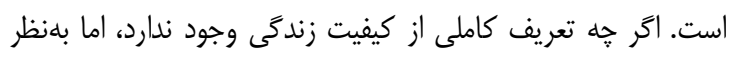

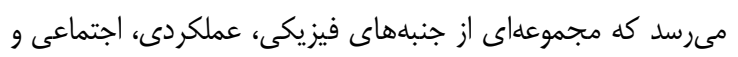

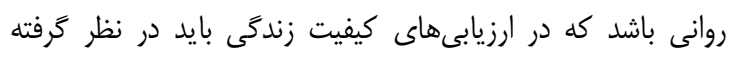

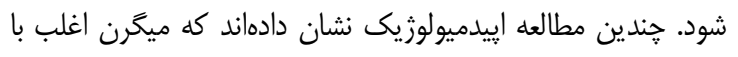

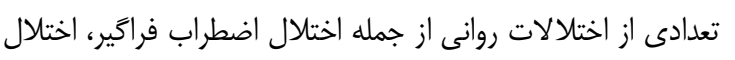

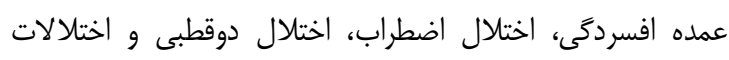

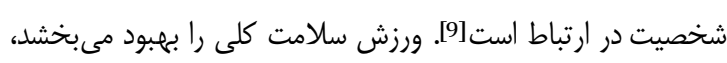

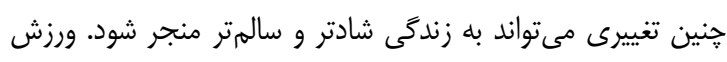

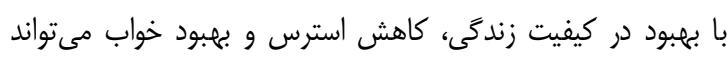

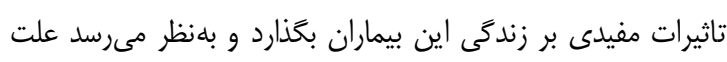

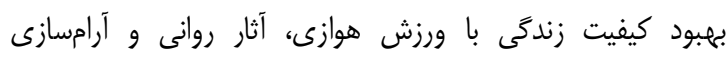

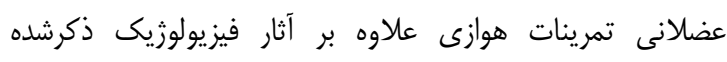

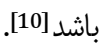

تاثيرات سودمند ورزش بر شاخصهاى ميكرن (دفعات، مدت و أندات

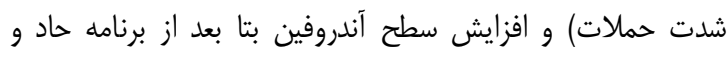

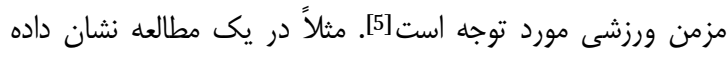

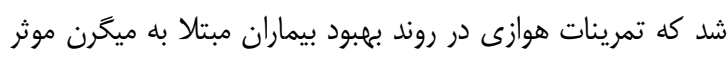

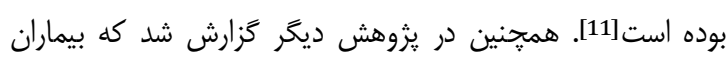

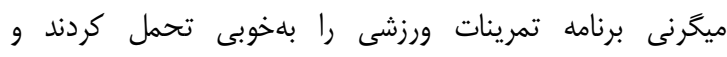

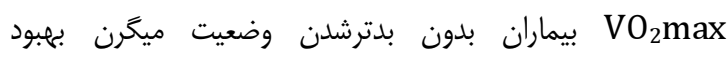

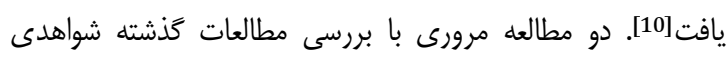

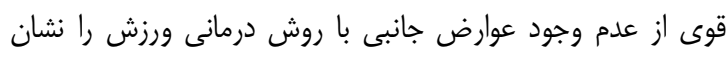

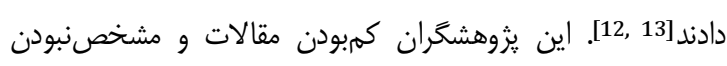

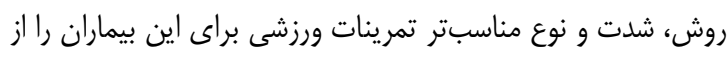

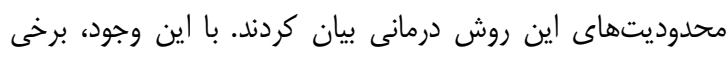

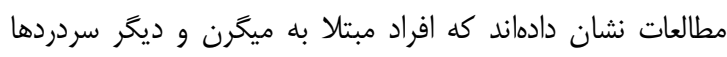

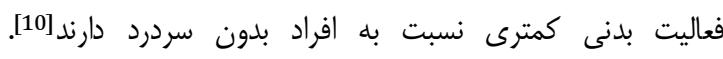

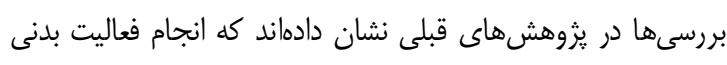

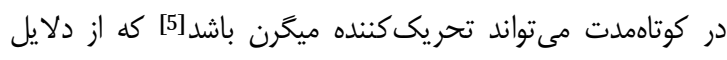

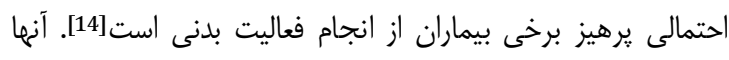

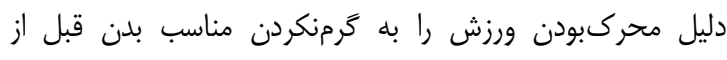

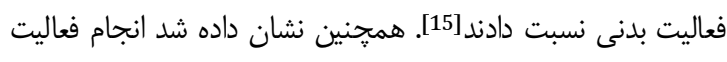

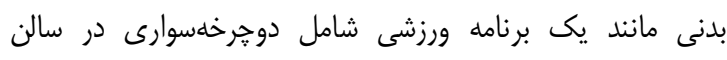

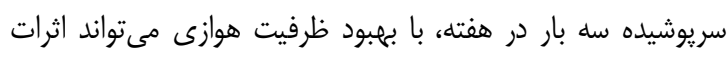

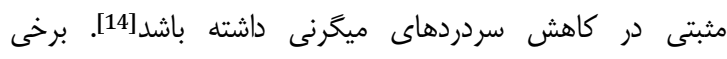

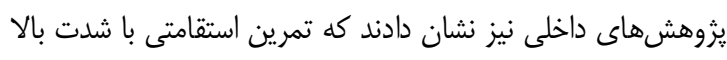

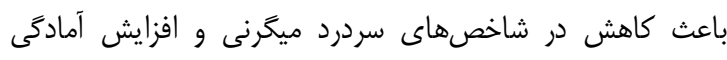
هوازى بيماران ميكرنى مىشود[9]. 


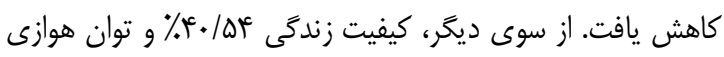

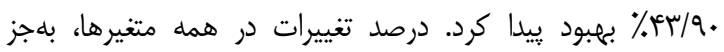

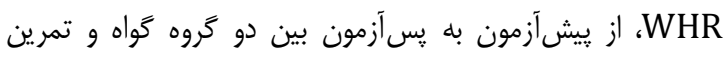

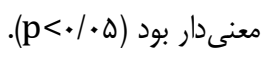

جدول T) مقايسه ميانكَين آمارى متغيرهاى يُورهش در هر دو كَروه كواه و

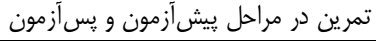

\begin{tabular}{|c|c|c|}
\hline $\begin{array}{c}\text { كروه تمرين } \\
\text { (•ا نفر) }\end{array}$ & 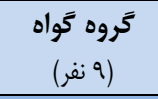 & متغيرها \\
\hline $\begin{array}{l}V T / r \varepsilon_{ \pm} \mid r / \cdot . \\
q Q / \Delta \Lambda \pm \mid r / V .\end{array}$ & $\begin{array}{l}q T / r \cdot \pm 1 \cdot / \cdot . \\
q T / r \pm 11 / k r\end{array}$ & 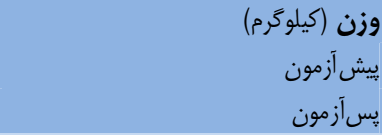 \\
\hline $\begin{array}{l}r N / T F \pm F / Q Y \\
r V / \backslash N \pm F / Q .\end{array}$ & $\begin{array}{l}r F / l \cdot \pm F / K^{E T} \\
r F / D \cdot \pm F / K^{C}\end{array}$ & يسيش آزآزهون توده بدن (كيلوَّرم بر متر مربع) \\
\hline $\begin{array}{l}r r / \cdot r \pm \varepsilon / F \Delta \\
r q / r \Lambda \pm r / r V\end{array}$ & $\begin{array}{l}r N / \Delta T^{r} \pm \varepsilon / \Lambda q \\
r N / T^{c} \cdot \pm V / Q T\end{array}$ & هيش \\
\hline $\begin{array}{l}\cdot \mid \Lambda T \pm \cdot / \cdot r \\
\cdot|\Lambda| \pm \cdot / \cdot r\end{array}$ & $\begin{array}{l}\cdot|\Lambda| \pm \cdot / \cdot q \\
\cdot \mid \Lambda \mathrm{N}_{ \pm \cdot / \cdot \Lambda}\end{array}$ & 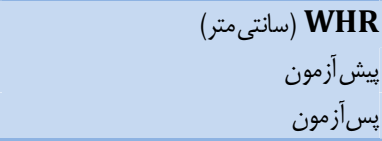 \\
\hline $\begin{array}{l}V / \Delta \Delta \pm I / l . \\
r / T r \pm I / r \Lambda\end{array}$ & $\begin{array}{l}\text { (Vas } \\
\Delta / V \Delta \pm T / /)^{c} \\
\Delta / \cdot \varepsilon \pm V / \Delta \Delta\end{array}$ & شيش آزآزمون سردرد (مقياس تطابق ديدارى يا \\
\hline 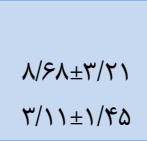 & $\begin{array}{l}1 \cdot / \Delta I \pm F / A r \\
N / \& T \pm r / Q \&\end{array}$ & 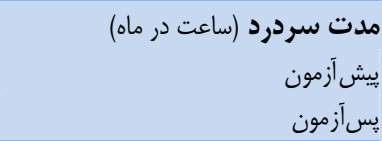 \\
\hline $\begin{array}{l}N / I \pm r / I F \\
r / \cdots \pm I / I\end{array}$ & 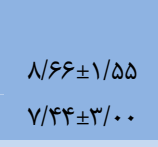 & 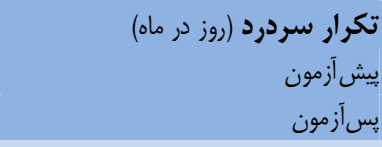 \\
\hline $\begin{array}{l}r q / F \pm r / l Q \\
r q / F r \pm r / r r\end{array}$ & 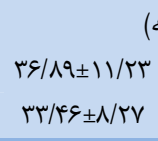 & 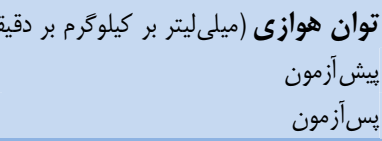 \\
\hline $\begin{array}{l}\varepsilon q / \cdot \xi \pm \varepsilon / \cdot \Delta \\
r q / 4 \cdot \pm r / 11\end{array}$ & $\begin{array}{l}\varepsilon \psi / \cdot \pm \Delta / \Delta F \\
\varepsilon \psi / \cdot \varepsilon \pm \Delta / \wedge \Delta\end{array}$ & 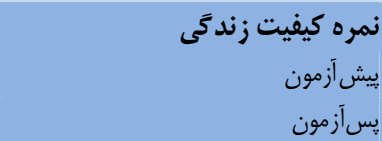 \\
\hline
\end{tabular}

بحث

يافتههاى بروهش حاضر نشان داد كه تمرينات MIA باعث كاهش

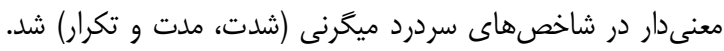

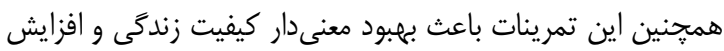

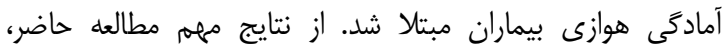

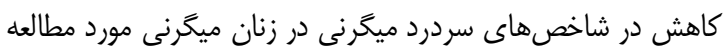

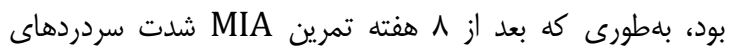

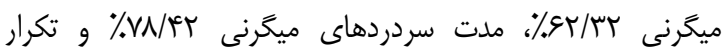

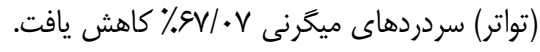

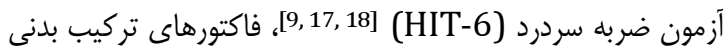
شامل؛ قد، سن، جنس، وزن و درصد جربى توسط دستخاه (BMI) (Zuse 9.9) composition

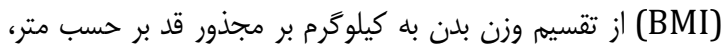

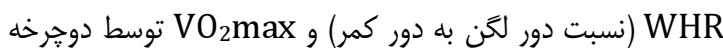

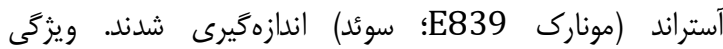

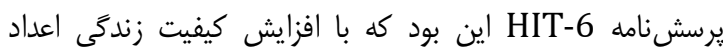

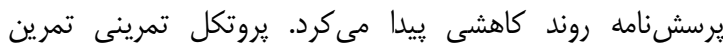

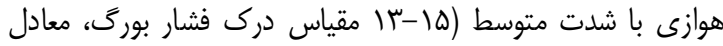

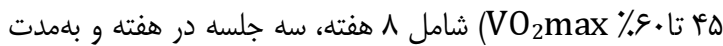

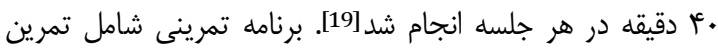
اصلى (دويدن)، •ا دقيقه گرمكردن و •ا دقيقه سردكردن بود

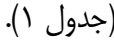

\begin{tabular}{|c|c|c|c|c|c|c|c|}
\hline هفته & هفته & شفته & هنته & هُهته & هفته & دفته & هفته \\
\hline \multirow{3}{*}{ f. } & & & & \multicolumn{4}{|c|}{ يانكين مدتزمان تمرين (دقيقه) } \\
\hline & f. & r. & מש & $r$. & $r \Delta$ & $r$. & 1. \\
\hline & & & & \multicolumn{4}{|c|}{ ميانَين شدت (درى فشار بورگ) } \\
\hline 10 & 10 & 10 & 10 & 10 & If & IT & 1. \\
\hline
\end{tabular}

براى رسم جداول و ارايه ميانگين آمارى از آمار توصيفى استفاده

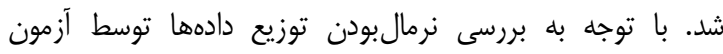

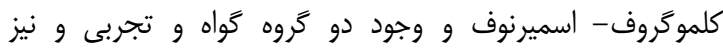

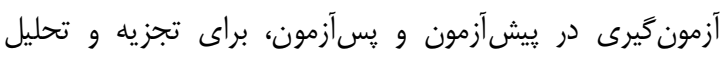

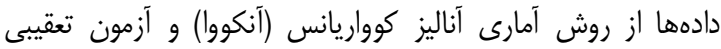

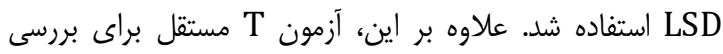

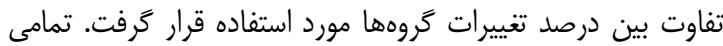
عمليات آمارى از طريق نرمافزار SPSS 21 انجام شد.

يافتهها ميانكين سنى آزمودنىها در كَروه كَواه تمرين

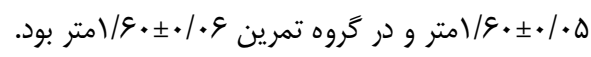

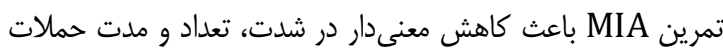

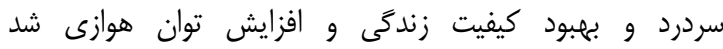

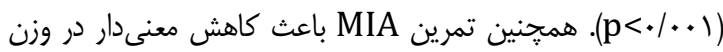

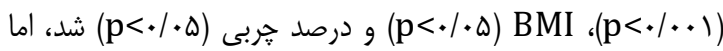

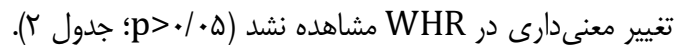

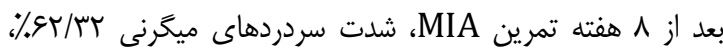

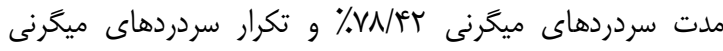

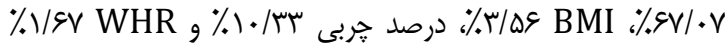

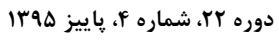

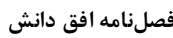




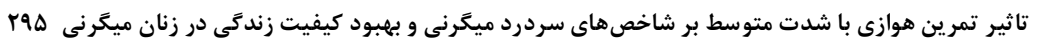

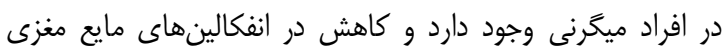

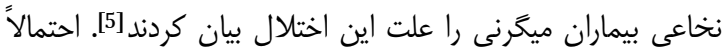

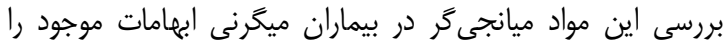

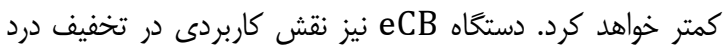

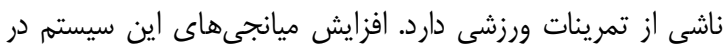

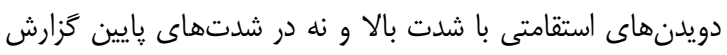

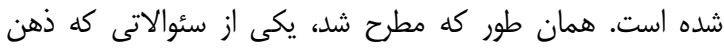

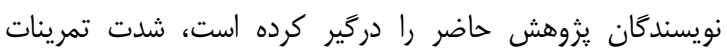

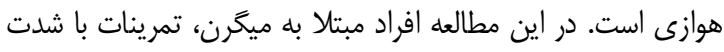


دستخاه eCB د دور از ذهن نيست.

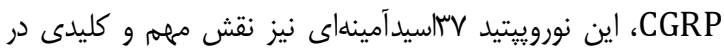

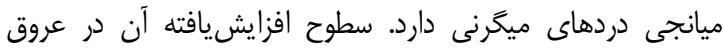

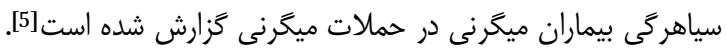

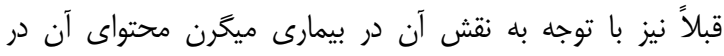

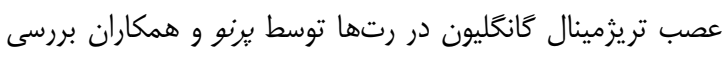

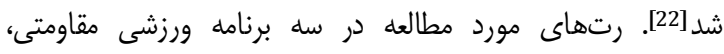

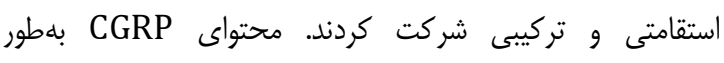

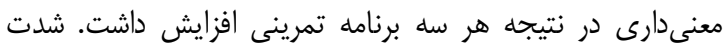

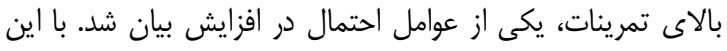

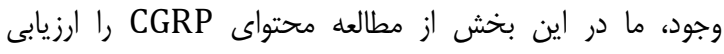

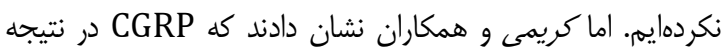

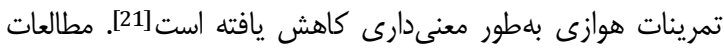

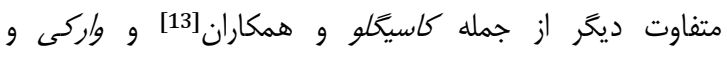

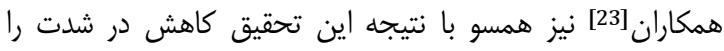
نشان دادند.

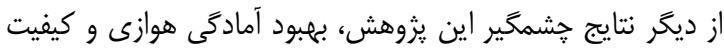

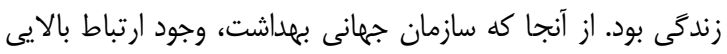

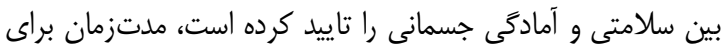

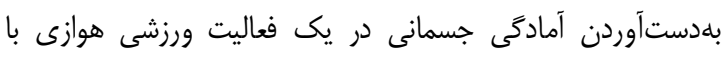
شدت متوسط •ما دقيقه در هفته و براى تمرينات هوازى دانى با شدات

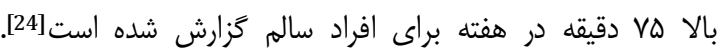

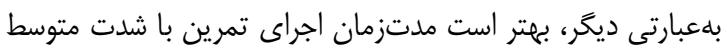

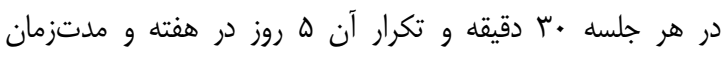

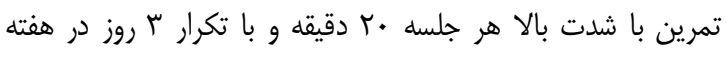

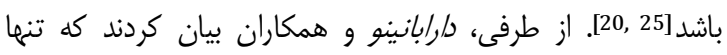

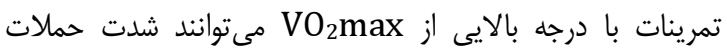

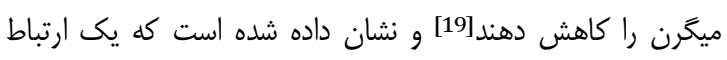

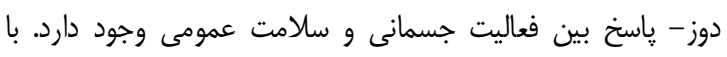

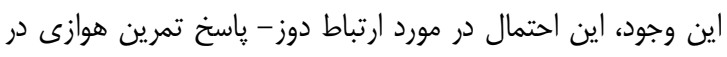

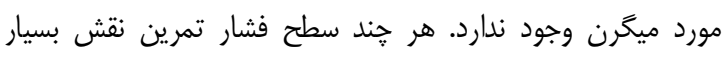

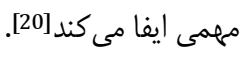

اغلب مطالعات كذنشته، مشابه اين دادههاى آمارى را تَزارش

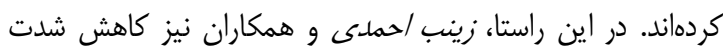

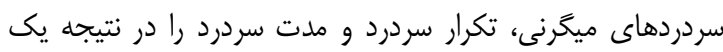

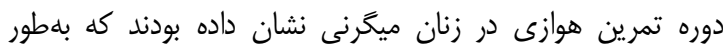

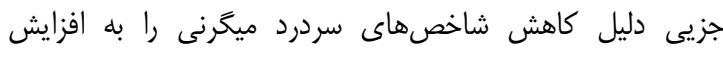

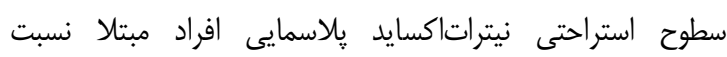

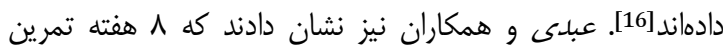

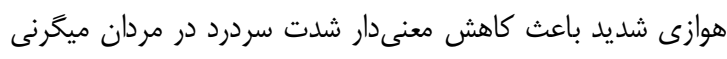

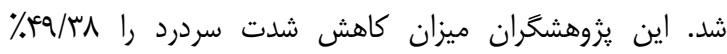

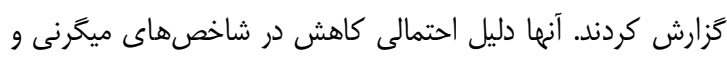

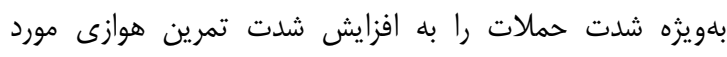

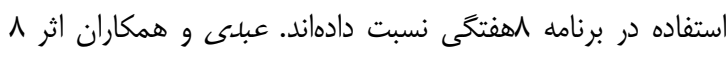

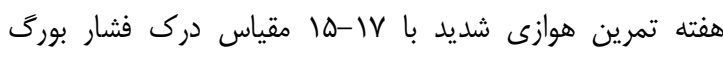

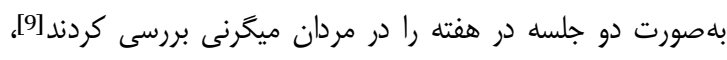

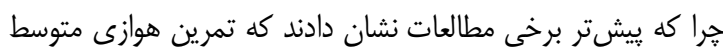

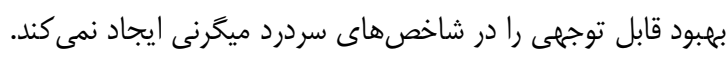

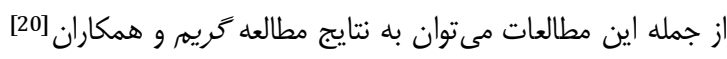

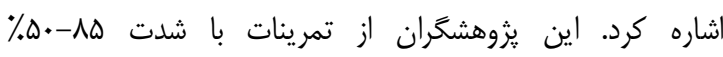

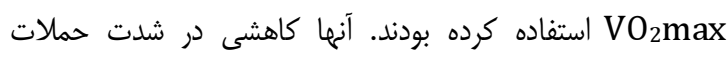
ميكَرن تزارش نكردند.

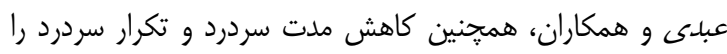

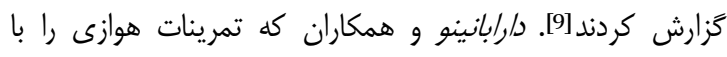

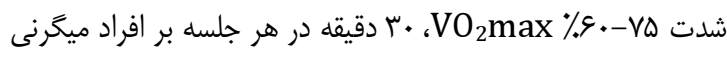

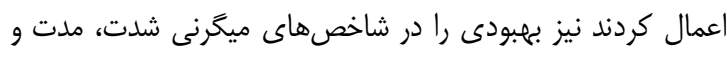

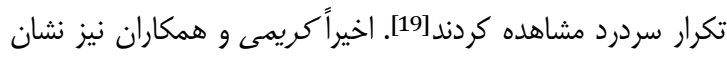

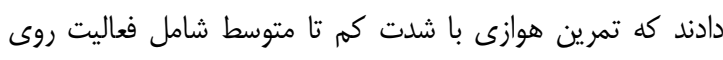

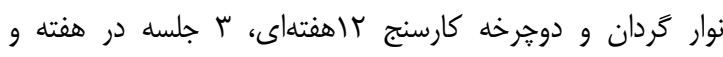

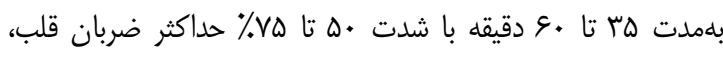

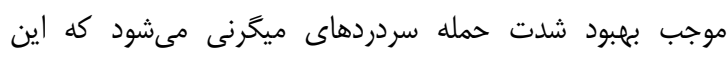

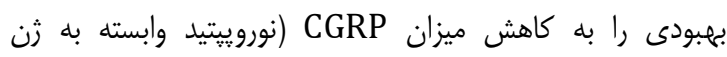
كلسىتونين) نسبت دادهاند[21].

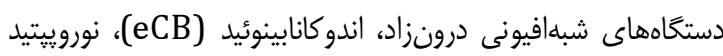

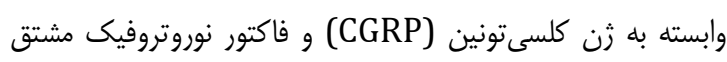

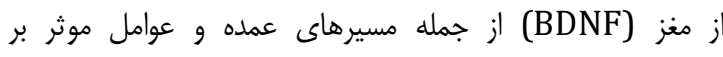

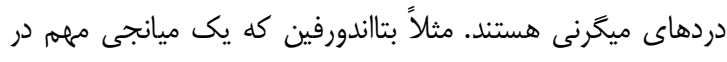

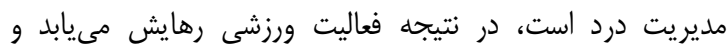

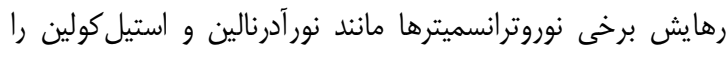



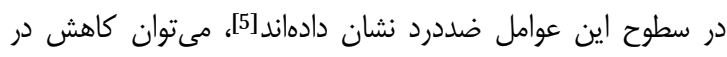

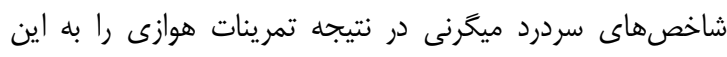

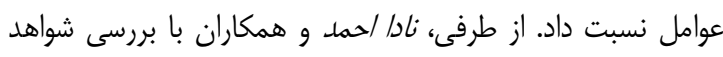

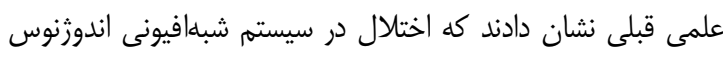


آكَاهى ناكافى و نحَرشهاى منفى قبلى مانند محركدانستن فعاليت

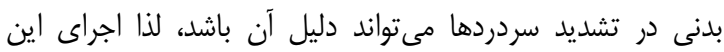

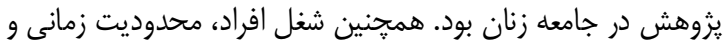

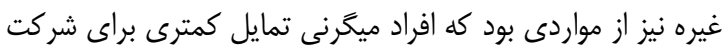

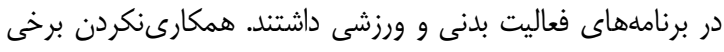

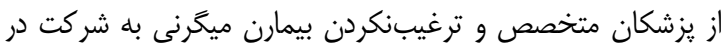
برنامهاى ورزشى نيز نقش مهمى در ايجاد اين محدوديتهان

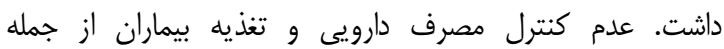

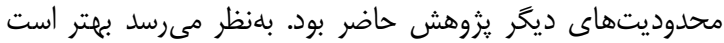

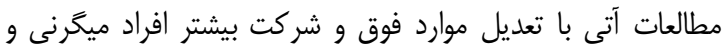

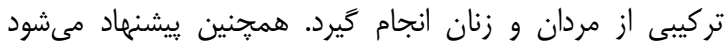

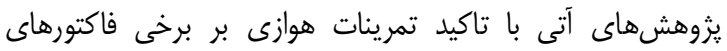

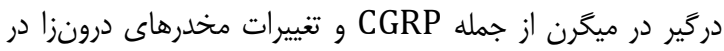

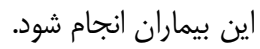

\section{نتيجه كَيرى}

تمرين هوازى با شدت متوسط در كاهش شاخصهاى سردرد ميكرنى و بهبود كيفيت زندگى و آمادگى هوازى در زنان مبتلا به به ميكَرن موثر است.

تشكر و قدردانى: از تمامى بيماران ميكرنى كه در اين يثرهش

$$
\text { شركت نمودند، تشكر و قدردانى مى شود. }
$$

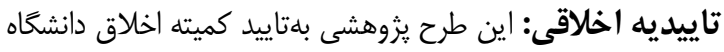

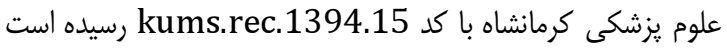
و با شماره كارآزمايى بالينى ثبت شده است.

تعارض منافع: موردى توسط نويسندًان اعلام نشده است.

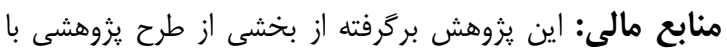

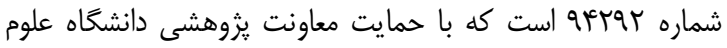

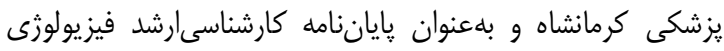

$$
\text { ورزشى انجام شده است. }
$$

\section{منابع}

1- Schwartz JS, Song P, Blitzer A. Headache, in therapeutic uses of botulinum toxin. In: Cooper G, editor. Therapeutic Uses of Botulinum Toxin. New Jersey: Humana Press; 2007. pp. 91-108.

2- Durham Durham PL, Garrett FG. Neurological mechanisms of migraine: potential of the gap-junction modulator tonabersat in prevention of migraine. Ceph. 2009;29(Suppl 2):1-6.

3- Lipton RB, Bigal ME. Migraine and other headache disorders: Informa health care. London: Taylor and Francis; 2006. pp. 45-120.

4- Eftekhari S, Edvinsson L. Possible sites of action of the new calcitonin gene-related peptide receptor

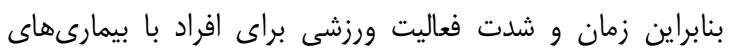

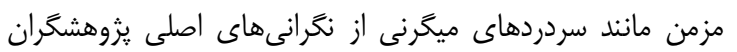

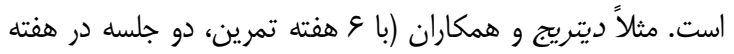

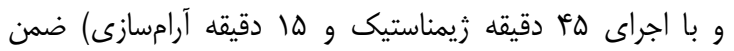

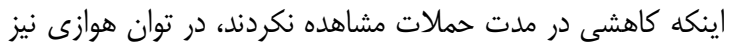

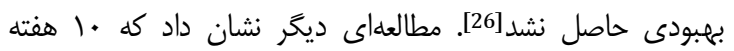

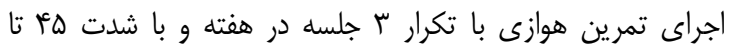
\% \%

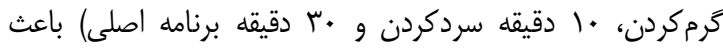

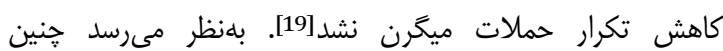

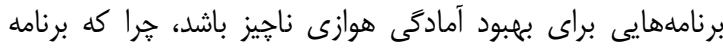

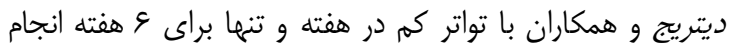

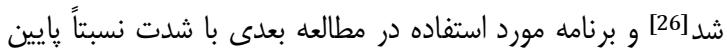

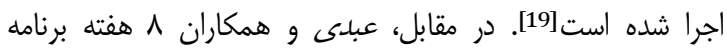

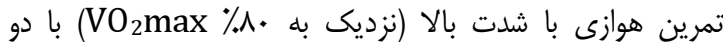

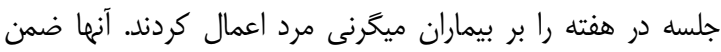

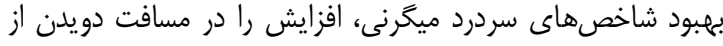

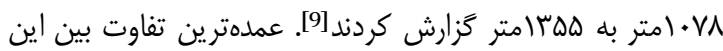

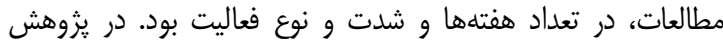
حاضر مدت مناسب تمرينات 1 هفته، تواتر جلسات تمرينى در هن هنته

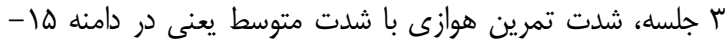

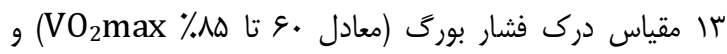

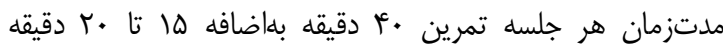

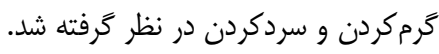

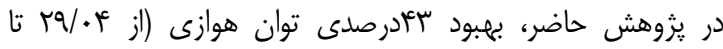

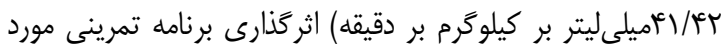

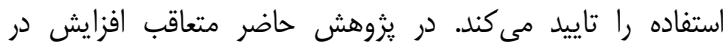

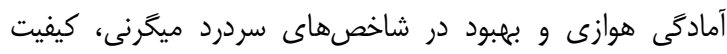

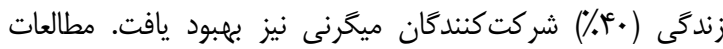

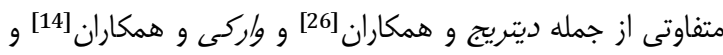

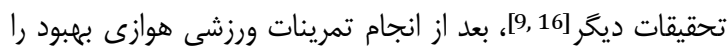

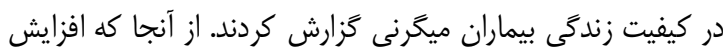

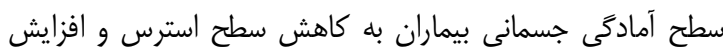

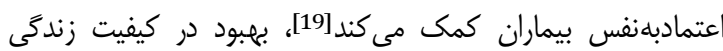

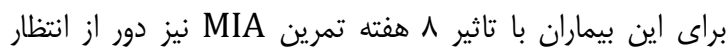

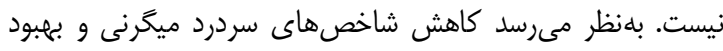

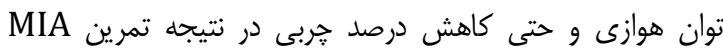

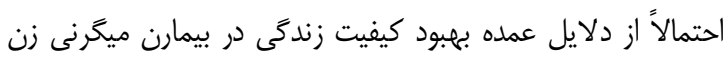

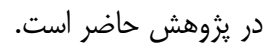
يزوهشكران براى انجام مطالعه حاضر با محدوديتهايى مواجه

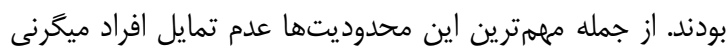

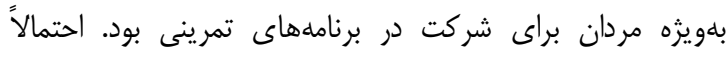


تاثير تمرين هوازى با شدت متوسط بر شاخصهاى سردرد ميكرنى و بهبود كيفيت زندكى در زنان ميگرنى

15- Kelman L. The triggers or precipitants of the acute migraine attack. Ceph. 2007;27(5):394-402.

16- Ahmadi Z, Taedibi V, Razaziyan N. The effect of 8week aerobic exercise on migraine headache indices and blood nitric oxide level in women with migraine. Exerc Physiol. 2015;7(26):33-50. [Persian]

17- Zandifar A, Banihashemi M, Haghdoost F, Masjedi SS, Manouchehri N, Asgari F, et al. Reliability and validity of the persian hit-6 questionnaire in migraine and tensiontype headache. Pain Pract. 2014;14(7):625-31.

18. Yang $M$, Rendas-Baum $R$, Varon S, Kosinski M. Validation of the headache impact test (HIT- $6^{\text {TM }}$ ) across episodic and chronic migraine. Ceph. 2011;31(3):357-67. 19- Darabaneanu S, Overath CH, Rubin D, Lüthje S, Sye W, Niederberger U, et al. Aerobic exercise as a therapy option for migraine: A pilot study. Int J Sports Med. 2011;32(6):455-60.

20- Sauro KM, Becker WJ. The stress and migraine interaction. Headache. 2009;49(9):1378-86.

21- Karimi F, Minasian V, Marandi SM, Chitsaz A. The Effect of a selected aerobic exercise on some physiological factors in patients with migraine. J Sport Biosci. 2015;7(2):329-45. [Persian]

22- Parnow AH, Gharakhanlou R, Eslami R. Effects of physical activity on calcitonin gene-related peptide content at trigeminal ganglion nerve in wistar rats. J Mazandaran Univ Med Sci. 2012;22(90):25-31. [Persian] 23- Varkey E, Cider A, Carlsson J, Linde M. Exercise as migraine prophylaxis: A randomized study using relaxation and topiramate as controls. Ceph. 2011;20(14):1428-38.

24- Leonardsson-Hellgren M, Gustavsson UM, Lindblad $\mathrm{U}$. Headache and associations with lifestyle among pupils in senior level elementary school. Scand J Prim Health Care. 2001;19(2):107-11.

25- Haskell WL, Lee IM, Pate RR, Powell KE, Blair SN, Franklin BA, et al. Physical activity and public health: updated recommendation for adults from the American College of Sports Medicine and the American Heart Association. Med Sci Sports Exerc. 2007;39(8):1423-34.

26- Dittrich SM, Günther V, Franz G, Burtscher M, Holzner B, Kopp M. Aerobic exercise with relaxation: Influence on pain and psychological well-being in female migraine patients. Clin J Sport Med. 2008;18(4):363-5. antagonists. Ther Adv Neurol Disord. 2010;3(6):369-78. 5- Hindiyeh NA, Krusz JC, Cowan RP. Does exercise make migraines worse and tension type headaches better? Curr Pain Headache Rep. 2013;17(12):380.

6- Thompson PD, Buchner D, Pina IL, Balady GJ, Williams MA, Marcus BH, et al. Exercise and physical activity in the prevention and treatment of atherosclerotic cardiovascular disease: A statement from the Council on Clinical Cardiology (subcommittee on exercise, rehabilitation, and prevention) and the council on nutrition, physical activity, and metabolism (subcommittee on physical activity). Circ. 2003;107(24):3109-16.

7- Scheef L, Jankowski J, Daamen M, Weyer G, Klingenberg M, Renner J, et al. An fMRI study on the acute effects of exercise on pain processing in trained athletes. Pain. 2012;153(8):1702-14.

8- Osün Narin S, Erbas D, Oztürk V, Idiman F. The effects of exercise and exercise-related changes in blood nitric oxide level on migraine headache. Clin Rehabil. 2003;17(6):624-30.

9- Abdi A, Parnow A, Azizi M. Effect of eight weeks high intensity aerobic trainingon migrane headache indicators. Horizon Med Sci. 2014;20(3):133-8. [Persian] 10- Lockett DM, Campbell JF. The effects of aerobic exercise on migraine. Headache. 1992;32(1):50-4.

11- Totzeck A, Unverzagt S, Bak M, Augst P, Diener HC, Gaul C. Aerobic endurance training versus relaxation training in patients with migraine (ARMIG): study protocol for a randomized controlled trial. Trials. 2012;13(1):46.

12- Gil-Martínez A, Kindelan-Calvo P, Agudo-Carmona D, Muñoz-Plata R, López-de-Uralde-Villanueva I, La Touche $R$. Therapeutic exercise as treatment for migraine and tension-type headaches: A systematic review of randomised clinical trials. Rev Neurol. 2013;57(10): 43343. [Spanish]

13- Köseoglu E, Akboyraz A, Soyuer A, Ersoy AO. Aerobic exercise and plasma beta endorphin levels in patients with migrainous headache without aura. Ceph. 2003;23(10):972-6.

14- Varkey E, Cider A, Carlsson J, Linde M. A study to evaluate the feasibility of an aerobic exercise program in patients with migraine. Headache. 2009;49(4):563-70. 